\title{
Influencia del ángulo de iluminación solar y la altura de la toma de la imagen multiespectral sobre la estimación de biomasa de pasto kikuyo
}

\section{Influence of height and zenith angle of multispectral images on the estimation of kikuyo grass biomass}

\author{
William Posada-Asprilla ${ }^{1}$; Mario Fernando Cerón-Muñoz ${ }^{2}$
}

IIng. Agrícola. Universidad de Antioquia, Facultad de Ciencias Agrarias, Grupo de Investigación GaMMA. Medellín, Antioquia, Colombia; e-mail: william. posada1@udea.edu.co; Dhttp://orcid.org/0000-0002-7663-1592

ZZootecnista, M.Sc., Dr. Universidad de Antioquia, Facultad de Ciencias Agrarias, Grupo Investigación en GaMMA. Medellín, Antioquia, Colombia; e-mail grupogamma@udea.edu.co; Dhttp://orcid.org/0000-0002-7233-6625

Cómo citar: Posada-Asprilla, W.; Cerón-Muñoz, M.F. 2019. Influencia del ángulo de iluminación solar y la altura de la toma de la imagen multiespectral sobre la estimación de biomasa de pasto kikuyo. Rev. U.D.C.A Act. \& Div. Cient. 22(2):e1338. https://doi. org/10.31910/rudca.v22.n2.2019.1338

Artículo de acceso abierto publicado por Revista U.D.C.A Actualidad \& Divulgación Científica, bajo una licencia Creative Commons CC BY-NC 4.0

Recibido: Julio 18 de 2018

Aceptado: Septiembre 6 de 2019

Editado por: Ingeborg Zenner de Polanía

\section{RESUMEN}

El objetivo de este estudio fue analizar la influencia del ángulo cenital de iluminación solar (mañana, mediodía y tarde) y la distancia (entre 10 y $50 \mathrm{~m}$ ) de imágenes multiespectrales de praderas de kikuyo, para la estimación de biomasa. Se capturaron datos espectrales de 40 muestras y se les calculó el índice de vegetación normalizada (NDVI) y la biomasa del forraje de las áreas fotografiadas. La relación de la biomasa con el NDVi, se hizo mediante modelos aditivos generalizados. Se encontró que es posible predecir la cantidad de biomasa con imágenes tomadas al mediodía y las alturas de vuelo analizadas (con $\left.\mathrm{R}^{2}=0,99\right)$, indicando que el monitoreo de praderas puede incorporar información de sensores con bandas de rojo e infrarrojo cercano, tomadas entre las 12:00m y 1:00pm.

Palabras clave: agricultura de precisión; índices de vegetación; modelos aditivos generalizados; sensor remoto.

\section{ABSTRACT}

The objective of this study was to analyze the effect of solar zenith angle (morning, noon and afternoon) and the distance (between 10 and $50 \mathrm{~m}$ ) of the multispectral images of kikuyo grasslands for biomass estimation. Spectral data from 40 samples were captured and the normalized vegetation index (NDVI) and the forage biomass of the photographed areas were calculated. The relationship of the biomass with the NDVi was made using generalized additive models. It was found that it is possible to predict the amount of biomass with images taken at noon and the flight heights analyzed (with $\mathrm{R} 2=0.99)$, indicating that grassland monitoring can incorporate sensor information with red and near infrared bands taken between 12:00m and 1:00 pm.

Keywords: generalized additive models; precision farming; remote sensor; vegetation index. 


\section{INTRODUCCIÓN}

La creciente utilización de índices de vegetación (VI) calculados con datos de sensores remotos, específicamente de imágenes multiespectrales, obtenidas a partir de aeronaves no tripulados (UAV), podrían constituir una técnica para el monitoreo de la productividad de los pastizales (Christensen et al. 2003; Cáceres, 2016). Un VI, se puede definir como un parámetro calculado a partir de los valores de reflectancia a distintas longitudes de onda y que puede ser relacionada con parámetros fisiológicos y de crecimiento de la vegetación (Tucker, 1979; Gilabert et al. 1997).

La firma espectral de las plantas entre 400 a $700 \mathrm{~nm}$ correspondiente al espectro visible, donde la reflectancia está controlada por la concentración de pigmentos en la hoja, principalmente, clorofila y carotenoides (Gausman et al. 1973). Esta región, se caracteriza por baja reflectancia y transmitancia del espectro visible, dada la fuerte absorción por los pigmentos foliares, donde la clorofila absorbe el espectro visible alrededor de las bandas azul (B) y roja (R) y su reflectividad es baja, mientras que en la banda espectral verde $(G)$, se refleja con mayor fuerza (Meer \& Jong, 2001). En esta región del espectro, se produce la fotosíntesis, proceso que implica la adsorción de energía. La mayor reflectancia de la vegetación verde (cerca del 45 y 50\%), se da en el infrarrojo cercano (NIR), causada por la difusión que resulta de los índices de refracción del líquido intracelular y de los espacios intercelulares del mesófilo de la planta (Gausman, 1977), donde el mesófilo esponjoso es el responsable de la elevada reflectancia que se produce en este espectro.

Uno de los VI más usados es el índice de vegetación de diferencia normalizada (NDVI), el cual, es usado para estimar la cantidad, la calidad y el desarrollo de la vegetación (Gilabert et al. 1997; MenesesTovar, 2011). Según Rouse et al. (1974), el NDVI mide la relación entre la energía absorbida y emitida por las plantas mediante la medición de la intensidad de la radiación incidente reflejada de las bandas NIR y R; sin embargo, hay que tener en cuenta los factores que provocan distorsiones en la radiación medida, como es el ángulo cenital de iluminación solar, las condiciones atmosféricas y la distancia entre el cenit y el nadir del sensor al objeto (Bolaños et al. 2010).

El ángulo cenital de iluminación referido al ángulo que forma la línea entre el observador y el sol y la vertical afecta los valores del NDVI, principalmente, por la dispersión en la radiación reflejada, debido a la no difusión lambertiana de las cubiertas vegetales, donde las respuestas espectrales de las plantas presentan un comportamiento anisótropo variante durante el día (Kimes, 1983; Guyot, 1984). Además, el NDVI es un índice diseñado para una observación vertical y, por lo tanto, es sensible a la geometría de adquisición (Camacho de Coca et al. 2002). Las condiciones atmosféricas influencian la intensidad de radiación incidente sobre la vegetación y la difusión de la radiación reflectante por aerosoles en el ambiente (Schnetzler, 1981). Además, hay que tener en cuenta la distancia entre el cenit y el nadir (altura entre el sensor y el objeto), que determina la resolución espacial y permite discriminar los menores objetos en el área de estudio (Guyot, 1980; Blanco et al. 2009).
El objetivo de este trabajo fue analizar la influencia del ángulo cenital de iluminación solar y la altura de vuelo en datos espectrales UAV, para la estimación de biomasa de pasto kikuyo, a través del NDVI.

\section{MATERIALES Y MÉTODOS}

El estudio, se llevó a cabo en la finca La Montaña de la Universidad de Antioquia, a $6^{\circ} 26^{\prime} 46,18^{\prime \prime} \mathrm{N}, 75^{\circ} 32^{\prime} 43,11^{\prime \prime W}$ y una altitud de $2.500 \mathrm{~m}$ s.n.m. La especie de pasto predominante en el sitio de estudio es la gramínea Cenchrus clandestinus (Hochst. ex Chiov. Morrone), el suelo es un andisol, típico de la región, con una profundidad media de $25 \mathrm{~cm}$ de textura franco-arcillosa.

Se identificaron 40 puntos de muestreo en las praderas usando un marco de $0,5 \times 0,5 \mathrm{~m}$; el pasto alrededor del cuadrante fue cortado para marcar el área de muestreo y evitar la contaminación de la estructura del marco en los valores de reflactancia (Figura 1). En estos puntos, se tomaron imágenes espectrales de las bandas $\mathrm{R}$ (longitud y ancho de onda de 668 y $10 \mathrm{~nm}$, respectivamente) y NIR (longitud y ancho de onda de 840 y 40nm, respectivamente), con un sensor multiespectral Micasense RedEdge ${ }^{\circledR}$ acoplado a un UAV DJI Phantom 4.

Las imágenes, se tomaron en la mañana (am), mediodía (m) y tarde $(\mathrm{pm})$, entre las $8: 00$ y $9 \mathrm{am}, 12: 00 \mathrm{~m}$ y 1:00pm y 3:00 y 4:00pm, respectivamente y que corresponden a un ángulo cenital entre 22 y $36^{\circ}, 65$ y $63^{\circ}$ y 42 y $29^{\circ}$, respectivamente. Antes de cada vuelo, se tomó una imagen del panel reflectante de calibración (PRC) para la corrección radiométrica de las imágenes. Las misiones de vuelos fueron programadas usando Pix 4D capture ${ }^{\circledR}$. Las alturas de vuelo utilizadas en las misiones fueron a 10, 20,30,40 y 50m, a una velocidad de $6 \mathrm{~m} / \mathrm{s}$. Posteriormente, en cada punto, se cortó, a $10 \mathrm{~cm}$ del suelo, la biomasa verde (BV) y se pesó. También, se tomaron datos de temperaturas, de humedad relativa, de viento y de radiación solar, usando una estación meteorología Vantaje Pro 2.

Se empleó el software Pix4D mapper pro ${ }^{\circledR}$, para realizar el ensamblado de las imágenes en un mosaico y el cálculo de los valores de reflectancia. Los recortes de los puntos de muestreo en las imágenes y el NDVI, se realizaron usando las librerías rgdal (Bivand y Rowlingson, 2016) y raster (Hijmans, 2016) del software R-project (R Core Time, 2017). El NDVI, se calculó mediante la ecuación propuesta por Rouse et al. (1974):

$$
\mathrm{NDVI}=\frac{\mathrm{NIR}-\mathrm{R}}{\mathrm{NIR}+\mathrm{R}}
$$

Los análisis, se realizaron utilizando modelos aditivos generalizados (gam), con funciones suavizadas no paramétrizadas (splines de regresión cúbica), utilizando la librería mgcv (Wood, 2017), del software R-Project (R Core Time, 2017). Se evaluaron 10 modelos para cada horario (3) y altura (5), para un total de 150 modelos, en el que las variables se sometieron a diferentes interacciones y efectos de suavizado, para determinar el modelo que mejor relacione la BV con el NDVI, los cuales, presentaron la siguiente estructura: 
$\mathrm{R}$

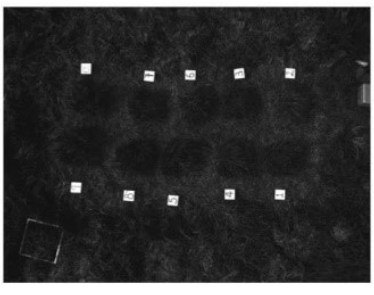

NIR

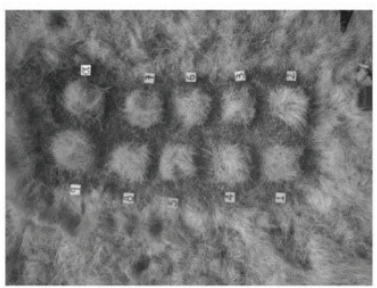

Foto aérea

Puntos de muestreo

Imágen espectral

Figura 1. Puntos de muestreo identificados en campo, utilizando un marco de $0,5 \times 0,5 \mathrm{~m}$ e imagen espectral de los puntos de muestreo de la banda roja $(\mathrm{R})$ y banda infrarroja cercana (NIR).

$$
\begin{aligned}
& Y_{i j}=\beta_{0}+s\left(N_{i}\right)+e_{i j} \\
& Y_{i j k l m n}=\beta_{0}+s\left(N_{i}\right)+s\left(T_{i}\right)+s\left(H_{k}\right)+s\left(S_{l}\right)+s\left(V_{m}\right)+e_{i j k l m n} \\
& Y_{i j k}=\beta_{0}+s\left(N_{i}\right)+s\left(T_{i j}\right)+e_{i j k} \\
& Y_{i j k}=\beta_{0}+s\left(N_{i}\right)+s\left(H_{i}\right)+e_{i j k} \\
& Y_{i j k}=\beta_{0}+s\left(N_{i}\right)+s\left(S_{i}\right)+e_{i j k} \\
& Y_{i j k}=\beta_{0}+s\left(N_{i}\right)+s\left(V_{i}\right)+e_{i j k} \\
& Y_{i j k l}=\beta_{0}+s\left(N_{i}\right)+s\left(T_{i j}, H_{k}\right)+e_{i j k l} \\
& Y_{i j k l}=\beta_{0}+s\left(N_{i}\right)+s\left(T_{j}, S_{k}\right)+e_{i j k l} \\
& Y_{i j k l}=\beta_{0}+s\left(N_{i}\right)+s\left(S_{j}, H_{k}\right)+e_{i j k l} \\
& Y_{i j k}=\beta_{0}+s\left(N_{i}\right)+s\left(I_{i}\right)+e_{i j k}
\end{aligned}
$$

Donde, Y es la biomasa verde en $\mathrm{g} / 0,25 \mathrm{~m} 2$, $\beta_{0}$ es el intercepto, $\mathrm{s}$ es la función suavizada de una variable o una combinación de dos variables, $\mathrm{N}$ es el índice de vegetación de diferencia normalizada, $\mathrm{T}$ es la temperatura en ${ }^{\circ} \mathrm{C}, \mathrm{H}$ es la humedad relativa en $\%$, S es la radiación solar en $\mathrm{w} / \mathrm{m} 2, \mathrm{~V}$ es el viento en $\mathrm{m} / \mathrm{s}$, I es el índice TSWH y e es el efecto residual.

El índice TSWH fue calculado con la ecuación propuesta por Steadman (1984):

$$
\text { TSWH }=-1,8+1,07 \mathrm{~T}+2,4 \mathrm{P}-0,92 \mathrm{~V}+0,044 \mathrm{~S}
$$

Donde, P es la presión de vapor en Kpa (50\% de humedad relativa es igual a $1,6 \mathrm{Kpa})$.

\section{RESULTADOS Y DISCUSIÓN}

El valor del intervalo NDVI obtenido varió entre 0,17 y 0,67; 0,68 y 0,82 ; y 0,18 y 0,60 , para cada horario am, $m$ y pm, respectivamente. Los mayores valores del NDVI, se presentaron en el horario $\mathrm{m}$, cuando la radiación solar presentó el mayor promedio $(844 \pm 216$ $\mathrm{w} / \mathrm{m}^{2}$ ), lo que concuerda con los resultados de Vercher et al. (2004), quienes observaron que el NDVI presenta una dispersión de sus valores del $70 \%$ por causa de las variaciones en las condiciones de observación o de iluminación.

En el estudio, se encontró que la temperatura, la humedad relativa, el viento y radiación solar y TSWH no tuvieron influencia en la variación de la $\mathrm{BV}(\mathrm{p}>0,05)$, únicamente, fue significativo el efecto del NDVI $(\mathrm{p}<0.01)$. Los análisis que surgieron del modelo 2 $\left(Y_{i j}=\beta_{0}+s\left(N_{i}\right)+e_{i j},\right)$, teniendo en cuenta las horas del día y la altura de vuelo, presentaron menor valor en el criterio de información bayesiano (BIC).

El resumen de los resultados obtenidos aparece en la tabla 1. Los modelos que mejor se ajustaron fueron: 2.6, 2.7, 2.8, 2.9 y 2.10, con $R^{2}$ superior a 0,98 ; los otros modelos presentaron un $R^{2}$, entre $0,00 y$ 0,30. Los mejores modelos corresponden a los datos obtenidos en el horario mediodía en alturas de 10, 20, 30, 40 y 50m. Por consiguiente, el efecto principal para establecer la relación del NDVI con la biomasa es la hora del día. Esto puede ser debido a que, a medida que aumenta la radiación solar, la actividad fotosintética en las plantas se intensifica, dado que existe una fuerte absorción por los pigmentos foliares, 
Tabla 1. Coeficiente de determinación y desviación explicada por los modelos de relación del NDVI, obtenido con imágenes multiespectrales, tomados a diferentes horas y alturas de vuelo, con la biomasa verde del pasto kikuyo.

\begin{tabular}{|c|c|c|c|c|}
\hline Modelo & Hora & Altura $(\mathbf{m})$ & $\mathbf{R}^{\mathbf{2}}$ & Des. explicada \\
\hline $\mathbf{2 . 1}$ & $8: 00-9: 00 \mathrm{am}$ & 10 & 0,30 & 0,31 \\
\hline $\mathbf{2 . 2}$ & $8: 00-9: 00 \mathrm{am}$ & 20 & 0,11 & 0,14 \\
\hline $\mathbf{2 . 3}$ & $8: 00-9: 00 \mathrm{am}$ & 30 & 0,00 & 0,00 \\
\hline $\mathbf{2 . 4}$ & $8: 00-9: 00 \mathrm{am}$ & 40 & 0,14 & 0,16 \\
\hline $\mathbf{2 . 5}$ & $8: 00-9: 00 \mathrm{am}$ & 50 & 0,11 & 0,13 \\
\hline $\mathbf{2 . 6}$ & $12: 00 \mathrm{~m}-1: 00 \mathrm{pm}$ & 10 & $\mathbf{0 , 9 8}$ & $\mathbf{0 , 9 9}$ \\
\hline $\mathbf{2 . 7}$ & $12: 00 \mathrm{~m}-1: 00 \mathrm{pm}$ & 20 & $\mathbf{0 , 9 8}$ & $\mathbf{0 , 9 9}$ \\
\hline $\mathbf{2 . 8}$ & $12: 00 \mathrm{~m}-1: 00 \mathrm{pm}$ & 30 & $\mathbf{0 , 9 9}$ & $\mathbf{0 , 9 8}$ \\
\hline $\mathbf{2 . 9}$ & $12: 00 \mathrm{~m}-1: 00 \mathrm{pm}$ & 40 & $\mathbf{0 , 9 9}$ & $\mathbf{0 , 9 9}$ \\
\hline $\mathbf{2 . 1 0}$ & $12: 00 \mathrm{~m}-1: 00 \mathrm{pm}$ & 50 & $\mathbf{0 , 9 9}$ & $\mathbf{0 , 9 9}$ \\
\hline $\mathbf{2 . 1 1}$ & $3: 00-4: 00 \mathrm{pm}$ & 10 & 0,25 & 0,34 \\
\hline $\mathbf{2 . 1 2}$ & $3: 00-4: 00 \mathrm{pm}$ & 20 & 0,00 & 0,00 \\
\hline $\mathbf{2 . 1 3}$ & $3: 00-4: 00 \mathrm{pm}$ & 30 & 0,00 & 0,00 \\
\hline $\mathbf{2 . 1 4}$ & $3: 00-4: 00 \mathrm{pm}$ & 40 & 0,27 & 0,30 \\
\hline $\mathbf{2 . 1 5}$ & $3: 00-4: 00 \mathrm{pm}$ & 50 & 0,00 & 0,02 \\
\hline & & & & \\
\hline
\end{tabular}

principalmente la clorofila, que absorbe con fuerza el espectro visible alrededor de las bandas $B$ y $R$, mientras que en la banda verde $(G)$, se refleja con mayor fuerza (Meer \& Jong, 2001).

En las horas del mediodía, el sol se acerca a la posición vertical (cenit), alcanzando una mayor altura solar, por lo que se genera una disminución en el área irradiada con energía constante y menor longitud del recorrido del rayo solar (Bach \& Franch, 2004), dando lugar a menores pérdidas por dispersión y al aumento en las reflectancias, cuando las mediciones se realizan en la misma dirección que la radiación entrante (Guyot, 1990). Durante los horarios am y pm, se presenta un efecto de sombra en la vegetación, el cual, está dado por el ángulo cenit de iluminación solar y la densidad foliar de las pasturas. Este efecto causa variaciones en las direcciones de la radiación reflejada, formando una cortina, que disminuye la intensidad de radiación a ciertas áreas foliares (Camacho de Coca et al. 2002).

Las resoluciones espaciales obtenidas por el sensor a los 10, 20, 30, 40 y $50 \mathrm{~m}$ de altura fueron $0,68,1,36,2,05,2,73$ y $3,41 \mathrm{~cm} /$ pixel, el cual, no fue un efecto influyente en los modelos 2.6, 2.7, 2.8, $2.9 \mathrm{y}$ 2.10 , principalmente, por la alta resolución manejada por el sensor (en $50 \mathrm{~m}$, se obtienen $3,4 \mathrm{~cm}$ por pixel), lo que permite realizar diferencias de las coberturas y los materiales en el sitio estudiado, a diferencia de los sensores en estaciones espaciales, como Landsat 8 y Sentinel 2, cuyas resoluciones espaciales son de 30 y $10 \mathrm{~m}$, respectivamente (Roy et al. 2014; Malenovský et al. 2012). Estos presentan dificultades, cuando se realizan estudios en pequeñas áreas, como es el caso de los potreros de pastos dedicados a producción de leche, en donde las áreas no sobrepasan los $5.000 \mathrm{~m}^{2}$.

La figura 2 muestra la relación entre la biomasa verde del área de muestreo y el NDVI obtenida con el modelo 2,9, donde se observa una variación del índice a medida que aumenta la biomasa $(\mathrm{p}<0,05)$. De igual forma, estudios como los de Escribano Rodríguez \& Hernández Díaz-Ambriona (2013) y Barrachina et al. (2010) propusieron una estimación de biomasa vegetal a través del NDVI, con $\mathrm{R}^{2}$ de 0,89 y 0,90 , respectivamente. Por el contrario, Mutanga \& Skidmore (2004) encontraron que el NDVI presentó un rendimiento bajo en la estimación de biomasa con $\mathrm{R}^{2}$, de 0,26 . Se registró que la diferencia entre los modelos obtenidos en el horario del medio día es mínima, por lo que la altura de vuelo del UAV recomendada por el estudio para la toma de las imágenes que evite obstáculos en las praderas, puede ser $50 \mathrm{~m}$.

El ángulo de iluminación solar afecta la cantidad de energía reflectante que llega al sensor, producto de las diferentes direcciones de dispersión de la radiación, siendo el mediodía el momento en el que la energía es reflejada en la misma dirección de llegada (menor ángulo entre la energía incidente y la reflejada), además de presentar los mayores valores de iluminación solar, debido a menores pérdidas por absorción y reflexión, lo que permite mejores imágenes.

El NDVI calculado al mediodía presentó menores desviaciones en la estimación de la biomasa de pasto kikuyo. Por consiguiente, puede 


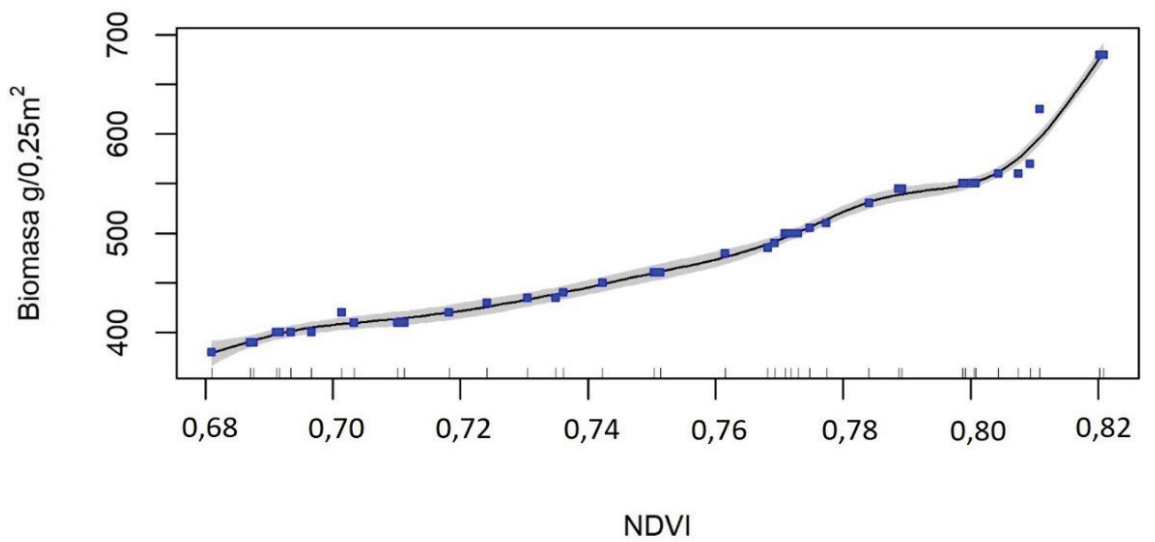

Figura 2. Relación entre el índice de vegetación de diferencia normalizada (NDVI) y la biomasa verde del pasto kikuyo.

ser incorporado en programas de evaluación de la productividad de los pastos.

Agradecimientos: Al proyecto "Fortalecimiento de la producción de la cadena láctea del distrito Norte Antioqueño", convenio No 2012AS180031 firmado entre La Secretaría de Agricultura y Desarrollo Rural del Departamento de Antioquia, la Universidad Nacional de Colombia (sede Medellín) y la Universidad de Antioquia. Conflicto de intereses: El manuscrito fue ejecutado, preparado y revisado con la participación de todos los autores, quienes declaramos que no existe conflicto de intereses que ponga en riesgo la validez de los resultados presentados. Financiación: Se recibió el apoyo del Sistema General de Regalías SGR y del Comité para el desarrollo de la investigación- CODI (Estrategia para la Sostenibilidad del grupo GaMMA)

\section{REFERENCIAS}

1. BACH, J.; FRANCH, J. 2004. La enseñanza del Sistema SolTierra desde la perspectiva de las ideas previas. Enseñ. cienc. Tierra. 12(3):302-312.

2. BARRACHINA, J.; CRISTOBAL, R.; TULLA, P.; ANTONI, F. 2010. Los recursos ganaderos en los sistemas extensivos de la montaña Pirenaica Catalana: aproximación al cálculo de la producción de biomasa herbácea mediante el uso de la teledetección. Ser. Geogr. 16:35-49.

3. BIVAND, R.; ROWLINGSON, B. 2016. rgdal: Bindings for the Geospatial Data Abstraction Library. R package version 1.2-5.

4. BLANCO, E.; BONET, J.A.; EIZAGUIRRE, M. 2009. Uso de imágenes satélite Landsat para la detección de rodales de Pinus nigra Arn y Pinus sylvestris L. afectados por escolítidos. Instituto Nacional de Investigación y Tecnología Agraria y Alimentaria (INIA). Invest. Agrar. Sist. Recur. For. 18:264-275.
5. BOLAÑOS, G.; MARTÍN, A.; PAZ, P.F. 2010. Modelación general de los efectos de la geometría de iluminación-visión en la reflectancia de pastizales. Rev. Mex. Cienc. Pecuarias. 1(4):349-361.

6. CÁCERES, J.D. 2016. Análisis del comportamiento temporal de la biomasa en pastos del Departamento de Olancho en el contexto del cambio global - fase II. Ciencias Espaciales. 7(1):97-114.

7. CAMACHO DE COCA, F.; GARCÍA-HARO, FJ.; GILABERT, M.A.; MELIÁ, J. 2002. La anisotropía de la BRDF: Una nueva signatura de las cubiertas vegetales. Rev. Teledetec. 18:29-46.

8. CHRISTENSEN, L.; COUGHENOUR, M.B.; ELLIS, J.E.; CHEN, Z. 2003. Sustainability of Inner Mongolian grasslands: application of the Savanna model. J. Range Managem. 56:319-327. http://doi.org/10.2307/4004034

9. ESCRIBANO RODRÍGUEZ, J.A.; HERNÁNDEZ DÍAZAMBRIONA, C.G. 2013. Estimación de la producción de pastos en Dehesas por índices de vegetación. En: Memorias Congreso: Los pastos: nuevos retos, nuevas oportunidades Badajoz, España. p.465-472.

10. GAUSMAN, H.W.; ALLEN, W.A.; WIEGAND, C.L.; ESCOBAR, D.E.; RODRIGUEZ, R.; RICHARDSON, A.J. 1973. The leaf mesophylls of twenty crops, their light spectra, and optical and geometrical parameters. USDA. Washington, D.C. Technical Bulletin. 1465. 68p.

11. GAUSMAN, H.W. 1977. Reflectance of leaf components. Remote Sensing of Environment. 6(1):1-9. https://doi. org/10.1016/0034-4257(77)90015-3 
12. GILABERT, M.A.; GONZÁLEZ-PIQUERAS, J.; GARCÍAHARO, J. 1997. Acerca de los índices de vegetación.Rev. Teledetec. 8:10-17.

13. GUYOT, G. 1980. Analysis of factors acting on the variability of spectral signatures of natural surfaces. In Proceedings International Symposium I.S.P. Hamburg. International Archives Photogrammetry 22:382-393.

14. GUYOT, G. 1984. Caractérisation spectrale des couverts végétaux dans le visible et le proche infrarouge, application a la télédétection. Bull. Soc. Franc. Photogram. et Télédétection. 95:5-22.

15. GUYOT, G. 1990. Optical properties of vegetation canopies. In: Steven, M D.; Clark, J.A. (eds). Application of Remote Sensing in Agriculture. Ed. Butterworths (Inglaterra). p.1944.

16. HIJMANS, R.J. 2016. Raster: Geographic Data Analysis and Modeling. R package version 2.5-8.

17. KIMES, D.S. 1983. Dynamics of directional reflectance factor distributions for vegetation canopies. Appl. Opt. 22:12641272.

18. MALENOVSKÝ, Z.; ROTT, H.; CIHLAR, J.; SCHAEPMAN, M.E.; GARCIA-SANTOS, G.; FERNANDES, R.; BERGER, M. 2012. Sentinels for Science: Potential of Sentinel-1, -2, and -3 missions for scientific observations of ocean, cryosphere, and land. Remote Sensing of Sentinels for science. 120:91-101. https://doi.org/10.1016/j. rse.2011.09.026

19. MEER, F.; JONG, S. 2001. Imaging Spectrometry. Remote Sensing and Digital Image Processing. Kluwe Academic Publishers. 4:111-197.

20. MENESES-TOVAR, C. 2011. El índice normalizado diferencial de la vegetación como indicador de la degradación del bosque. Unasylva. 238 62:39-46.

21. MUTANGA, O.; SKIDMORE, A.K. 2004. Narrow band vegetation indices overcome the saturation problem in biomass estimation. Int. J. Remote Sens. 25:3999-4014.
22. R CORE TEAM. 2017. R: A language and environment for statistical computing. R Foundation for Statistical Computing. Vienna, Austria.

23. ROUSE, J.W.; HAAS, R.H.; SCHELL, J.A.; DEERING, D.W. 1974. Monitoring Vegetation Systems in the Great Plains with ERTS, Third ERTS Symposium (Washington, DC: NASA). p.309-317.

24. ROY, D.P.; WULDER, M.A.; LOVELAND, T.R.; WOODCOCK.; C.E.; ALLEN, R.G.; ANDERSON, M.C.; HELDER, D.; IRONS, J.R.; JOHNSON, D.M.; KENNEDY, R.; SCAMBOS, T.A.; SCHAAF, C.B.; SCHOT'T, J.R.; SHENG, Y.; VERMOTE, E.F.; BELWARD, A.S.; BINDSCHADLER, R.; COHEN, W.B.; GAO, F.; HIPPLE, J.D.; HOSTERT, P.; HUNTINGTON, J.; JUSTICE, C.O.; KILIC, A.; KOVALSKYY, V.; LEE, Z.P.; LYMBURNER, L.; MASEK, J.G.; MCCORKEL, J.; SHUAI, Y.; TREZZA, R.; VOGELMANN, J.; WYNNE, R.H.; ZHU, Z. 2014. Landsat-8: science and product vision for terrestrial global change research. Remote Sensing of Environment. 145:154-172.

25. SCHNETZLER, C.C. 1981. Effect of sun and sensor geometry, canopy structure and density, and atmospheric condition on the spectral response of vegetation, with particular emphasis on accross-track pointing. In Proceedings International Colloguium Spectral Signatures of Objects in Remote Sensing. Avignon, France. 8-11 Sept. Les Collogues de VINRA. 5:509-520.

26. STEADMAN, R.G. 1984. A universal Scale of apparent temperature. J Clim. 23:1674-687.

27. TUCKER, C.J. 1979. Red and photographic infrared linear combinations for monitoring vegetation. Rev. Teledetec. 8(2):127-150.

28. VERCHER, A.; CAMACHO DE COCA, F; MELIÁ, J. 2004. Influencia de la geometría de adquisición en el NDVI. Rev. Teledetec. 21:95-99.

29. WOOD, S. 2017. Mixed GAM Computation Vehicle with GCV/AIC/REML smoothness estimation and GAMMs by REML/PQL. 